\title{
State of field measurement of facade sound insulation in buildings: evaluation of proficiency testing data according to ISO 140-5:1998
}

\author{
José Luis Martínez · José Alberto Trujillo · José Silván · Pedro Rosario · \\ Juan Sancho
}

\begin{abstract}
The ISO 140-5:1998 standard specifies the testing methods to evaluate the sound insulation in buildings and building elements for facades. Three rounds of a proficiency testing scheme for airborne sound insulation measurements have been performed according to the methods specified in the standard for a whole facade by using an external loudspeaker as the noise source. The adequacy of the intercomparison is guaranteed by means of the assessment of global precision criteria in compliance with the scope stipulated by the normative references in order to determine the reproducibility limits in a regulated measurement method. To evaluate the participant performance, the corresponding combined scores as composite indexes are calculated, so if this assessment is performed along a certain number of rounds, it will remark the participants that show an overall satisfactory performance by monitoring their achievement over time. Therefore, in order to establish a reference value in terms of precision level, an approach based on the development of a collaborative test in controlled conditions is proposed. The goal is to provide reliable and updated criteria for the evaluation of repeatability and reproducibility limits in this field, particularly in those scopes in which nowadays there is no normative reference or this is very poor. Furthermore, these prescribed values of precision should be verified
\end{abstract}

regularly, as well as the definition of practical conditions of application.

Keywords Sound insulation - Precision assessment . Combined performance scores · Collaborative study

\section{Introduction}

The series of ISO 140 standards named "acoustics-measurement of sound insulation in buildings and of building elements" describe the methods for measurement of airborne and impact sound insulation. In particular, the standard ISO 140-5:1998 "field measurements of airborne sound insulation of facade elements and facades" specifies the testing methods to evaluate the insulation of building facade elements and whole facades. For each method, testing procedures are established either using the broadband emission of a loudspeaker as an artificial sound source or using real noise available on the spot where the facade or facade element is located, such as road traffic, railway or even airport noise [1].

Since ISO/IEC 17025 requires testing laboratories to put in place external quality control procedures for monitoring the reliability of their measurements [2], the participation in proficiency testing schemes is the main mechanism to assess compliance.

In this context, the Spanish network of laboratories ACUSTILAB, which is set up within EUROLABESPAÑA organization, has established a project called AQUS with the aim of providing a tool for the objective verification of the technical competence by means of the development on an annual basis of an intercomparison scheme in the field of acoustics, noise and vibration measurements. 


\section{Methodology and limitations of the standard}

In order to satisfy this requirement, from the year 2011, three rounds of this proficiency testing scheme have been organized at the national level for measurements of airborne sound insulation of building facades, as stated by the scope of the standard UNE EN ISO 140-5:1999 (that will be replaced by the new standard ISO 16283-1). The tests were performed according to one of the methods specified by the standard, in particular for a whole facade by using an external loudspeaker as the noise source.

The parameter of the standardized sound level difference expressed in $\mathrm{dB}$ units and calculated according to ISO $717-1$ is $D_{\mathrm{Is}, 2 \mathrm{~m}, \mathrm{nT}}$ with measurements carried out in bands of a third of octave, in the frequency range $100-5000 \mathrm{~Hz}$, where the emission noise levels are ideally obtained from the direct field of the sound source [3]. In this way, the accuracy of the parameter depends on the difference between the measurements performed directly on the facade and the average of sound pressure level measured in five points located inside the building element itself.

Some difficulties found in measurements of facade sound insulation are related to the minor spatial sampling conducted in trials of facade and the use of a single source position. Therefore, both factors make noise levels far more stable, so when the same laboratory performs several replications, it achieves measurements with a relatively small repeatability value. Because of that, lack of spatial sampling, as well as the directional characteristics of noise sources used by different laboratories where compliance with the coverage of facade in many of the sources is ambiguous, makes the type of source and its position influence the final measurement [4]. These two additional factors, when different laboratories (and different equipments) perform the same test, cause that the reproducibility value is greater.

In the project AQUS, the description of the interlaboratory program is well documented, in which a series of planned stages related to the supervision, follow-up and control of the measurement items are considered. This monitoring phase is to assure both the stability of the scenario and the homogeneity of the sound levels intended for the evaluation of the test specimen [5]. These aspects are fully evaluated to guarantee the adequacy of the interlaboratory comparison by means of the assessment of certain global precision guidelines in compliance with the current scope, because no regulatory or prescribed values are available for reproducibility verification in this scope.

It is interesting to point out that the stability criteria were established before the execution of the measurements, so in the event of nonconforming control data in any frequency, the reason for that must be investigated and considered for future rounds. Since the stability of the scenario is evaluated and monitored by an accredited laboratory, it is allowed to declare that the conditions due to the site location remain unchanged over time.

\section{Statistical design and performance evaluation}

The protocol conducted in this proficiency test scheme outlines a statistical design not only intended for the analysis of the data but also for the evaluation of the proficiency testing results according to the standard ISO 5725-2 that describes the determination of the limits of repeatability and reproducibility in a regulated measurement procedure using classical statistical methods with outlier elimination [6].

As regards the implementation of the PT scheme AQUS, the statistical parameters published for the magnitudes object of study in the scope of the program are the following:

- assigned value in each frequency as consensus value from participants;

- estimate of the precision of the PT results in terms of repeatability and reproducibility limits; and

- standardized $z$-score taken as an assessment of the performance of the participation by each laboratory.

As for the determination of the assigned value, once discarded the outlier results, it is made in accordance with the established protocol by first calculating the arithmetical average of the four results reported by each one of the 14 participants, taken as consensus value for the number of non-excluded participants in each magnitude, the most appropriate measure of central tendency based on the distribution of the data of the participants, once verified the normality of them.

Then, to define the variability of the proficiency test results, it is first necessary to estimate values of the standard deviation under conditions of repeatability $\left(s_{r}\right)$ and the one under conditions of reproducibility $\left(s_{R}\right)$. These are applied to calculate the related parameters $r$ and $R$ according to ISO 5725-6

$r=2.8 s_{r}$

$R=2.8 s_{R}$

The parameters $r$ and $R$ may be called "critical differences" or "limits," as they describe the maximum difference of a pair of test results at which these can be considered equivalent at a probability level of $95 \%$.

The performance of each participant is evaluated by calculating the corresponding $z$-score, determined in accordance with: 
$z=\frac{x_{i}-X_{\mathrm{pt}}}{\sigma_{\mathrm{pt}}}$

where $x_{i}$ is the laboratory result, $X_{\mathrm{pt}}$ is the assigned reference value and $\sigma_{\mathrm{pt}}$ is the estimate of the standard deviation of the proficiency test results. In this way, results with a value $|z| \leq 2$ are considered as satisfactory; results with $|z| \leq 3$ are unsatisfactory; results with $2<|z|<3$ are considered questionable.

\section{Pitfalls to establish precision guidelines}

With respect to the assessment of the precision criteria for the proficiency test under ISO 140-5, regrettably there are no available any kind of regulatory values that can be used as a prescribed reference, unlike what happens in other parts of the standard ISO 140 [7]. In this way, the ISO 140-2 gives some indications to state the precision of the measurements carried out according to a certain test method that unfortunately cannot be applied to the current scope; hence, it is not possible to verify if the reproducibility values obtained during this proficiency testing scheme performed on ISO 140-5 are in accordance with a normative reference in terms of significant differences.

For this reason, in this paper, it is proposed the organization of a proficiency test to determine the aforementioned precision values by means of some operative steps that lead to the prescription of tentative values of reproducibility.

In order to evaluate the performance of the participants, since the global parameter $D_{\mathrm{ls}, 2 \mathrm{~m}, \mathrm{nT}}$ is determined from the individual measurements in bands of a third of octave in the frequency range $100-5000 \mathrm{~Hz}$, the corresponding combined performance scores as composite indexes are calculated on the basis of the determination of the SSZ values (sum of squares of $z$-scores). In addition, the $S S Z$ values obtained for each participant might be considered as a global quality indication that informs about the comprehensive laboratory participation in the considered range of frequencies by assessing the significant dispersions of the accumulated $z$-scores [8].

If this assessment is carried out along the three proficiency test rounds carried out up to date, since they are comparable enough, it might be possible to determine those participants that show an overall satisfactory performance in every scope of interest by monitoring their performance over time.

\section{Results and discussion}

Once performed, the three rounds in Fig. 1 are depicted the assigned values obtained from participant consensus in each frequency and each round, showing a high consistency among the values of the measurable parameter over time. So, it is worthy to affirm that the noise source is homogeneous enough, as it was previously stated in the methodology stage for monitoring the scenario.

Moreover, for a given round, the results calculated for each frequency as for the assigned value of $D_{1 \mathrm{~s}, 2 \mathrm{~m}, \mathrm{nT}}$ as well as the repeatability limit $(r)$ and the reproducibility limit $(R)$ would be expressed as in Table 1. For illustrative purposes, the values of $D_{1 \mathrm{~s}, 2 \mathrm{~m}, \mathrm{nT}, \mathrm{w}}$ (weighted standardized level difference according to ISO 717-1) and $D_{\mathrm{ls}, 2 \mathrm{~m}, \mathrm{nTA}}$ (global difference in $\mathrm{dBA}$ along $100-5000 \mathrm{~Hz}$ ) have been also included.

In summary, for measurements expressed as $D_{\mathrm{ls}, 2 \mathrm{~m}, \mathrm{nT}}$ parameter in the frequency range from 100 to $5000 \mathrm{~Hz}$, it is observed that the testing results throughout the rounds show in numbers that $89 \%$ of the participants present satisfactory results, whereas $7 \%$ can be considered to be questionable or unsatisfactory and only $4 \%$ of the participants have been detected as outliers. Although a slightly broader distribution was observed in the first PT round, we can say that the evolution of $z$-scores among the three rounds organized until now is considered both appropriate and representative in order to achieve the objectives of this program.

Consequently, after the application of the statistical protocol to compute the proficiency test results, the corresponding values of reproducibility standard deviation are calculated, as an indication of the reliability of the interlaboratory comparison. In Fig. 2, a comparison of the reproducibility limits $R$ obtained in each measuring frequency for each one of the three proficiency test rounds is depicted, in which the above-mentioned wider spread of $R$ values in the first round is obvious, although a quite similar distribution is found among the participants. In addition, because of the own characteristics of the measurement of facade sound insulation [9], the measurement uncertainty reported by the participants has been mostly greater in the low frequency range.

Considering that the degree of global variability over the rounds is not regarded as sufficiently appropriate to establish any precision guidelines on the basis of these results, it seems suitable to select just those laboratories which systematically have better performance over time with the aim of determining repeatability and reproducibility target values. Therefore, as a way of proving this result, the accumulated values SSZ for all participants are shown in Fig. 3, given the number of PT rounds in which every laboratory has taken part. According to the Chi-squared distribution, since the statistically acceptable $S S Z$ composite value is 28.9 (for the combination of 18 frequency values), as a whole, it is observed that the majority of participants show an adequate global performance. 


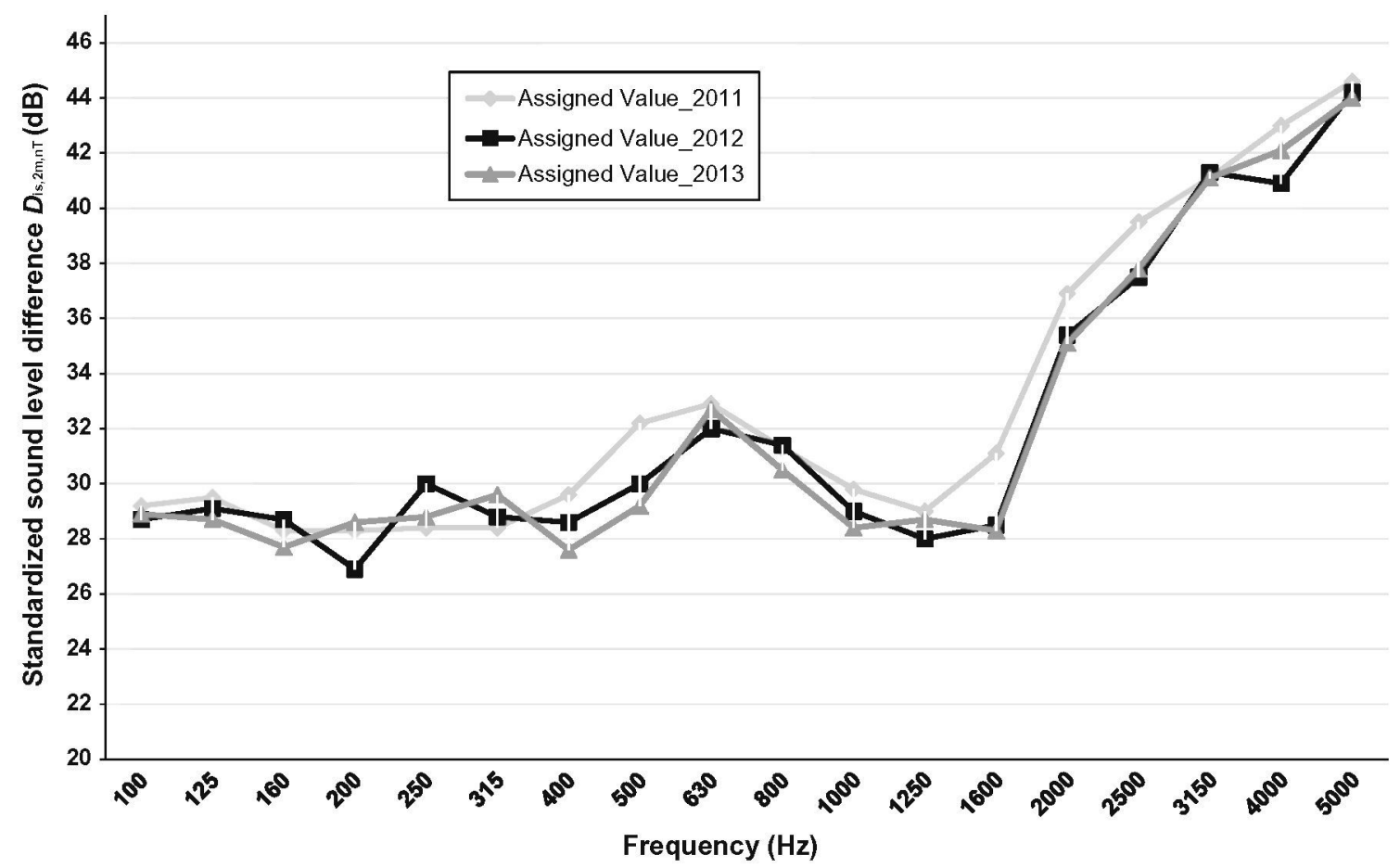

Fig. 1 Assigned values of facade sound insulation of buildings obtained in AQUS proficiency testing rounds

Table 1 Assigned values and precision limits in AQUS-2013 $(p=14$ participants)

\begin{tabular}{llll}
\hline $\begin{array}{l}\text { Frequency } \\
(\mathrm{Hz})\end{array}$ & $\begin{array}{l}\text { Assigned value } \\
(\mathrm{dB})\end{array}$ & $\begin{array}{l}\text { Repeatability } \\
\text { limit }(\mathrm{dB})\end{array}$ & $\begin{array}{l}\text { Reproducibility } \\
\text { limit }(\mathrm{dB})\end{array}$ \\
\hline 100 & 28.7 & 3.15 & 4.98 \\
125 & 29.1 & 2.59 & 6.50 \\
160 & 28.7 & 2.34 & 7.08 \\
200 & 26.9 & 3.22 & 7.61 \\
250 & 30.0 & 2.61 & 4.96 \\
315 & 28.8 & 2.29 & 5.43 \\
400 & 28.6 & 2.03 & 5.19 \\
500 & 30.0 & 1.67 & 5.18 \\
630 & 32.0 & 1.67 & 4.13 \\
800 & 31.4 & 1.56 & 3.32 \\
1000 & 29.0 & 1.45 & 5.57 \\
1250 & 28.0 & 1.71 & 6.19 \\
1600 & 28.5 & 1.48 & 6.18 \\
2000 & 35.4 & 1.56 & 3.24 \\
2500 & 37.5 & 1.83 & 8.04 \\
3150 & 41.3 & 1.21 & 2.90 \\
4000 & 40.9 & 1.25 & 5.49 \\
5000 & 44.2 & 0.89 & 3.88 \\
$D_{\mathrm{ls}, 2 \mathrm{~m}, \mathrm{nT}, \mathrm{w}}$ & 32.0 & 1.20 & 2.30 \\
$D_{\mathrm{ls}, 2 \mathrm{~m}, \mathrm{nTA}}$ & 30.6 & 1.06 & 2.62 \\
\hline & & &
\end{tabular}

Proposal for setting up reproducibility guidelines

Taking into consideration the variability of the previously exposed results obtained for the three AQUS proficiency test rounds developed up to now, we have decided to pose a simulation by selecting an arbitrary precision criteria based on the fact that the most reliable participants are selected according to the laboratories which systematically have achieved $S S Z$ values lower than 15.0 set as a target value that informs us of the ones which present the best achievement related to a sort of performance rating.

In this way, once computed all the $S S Z$ values obtained by the participants, we have selected the laboratories that meet this criterion on the measurement of $D_{\mathrm{ls}, 2 \mathrm{~m}, \mathrm{nT}}$ $(100-5000 \mathrm{~Hz})$ parameter, so four participants have been selected who complied with this empirical rule during their past involvement, as it is shown in Table 2. Therefore, these participants were considered as reference in terms of better precision execution and declared as expert laboratories for the successive calculations.

Therefore, in relation to our proposal based on the above results, some tentative $R$ values of the reproducibility limits would be established in accordance with the following prescribed magnitudes according to the consideration of the best performance of the most reliable participants in the Project AQUS [10]. 


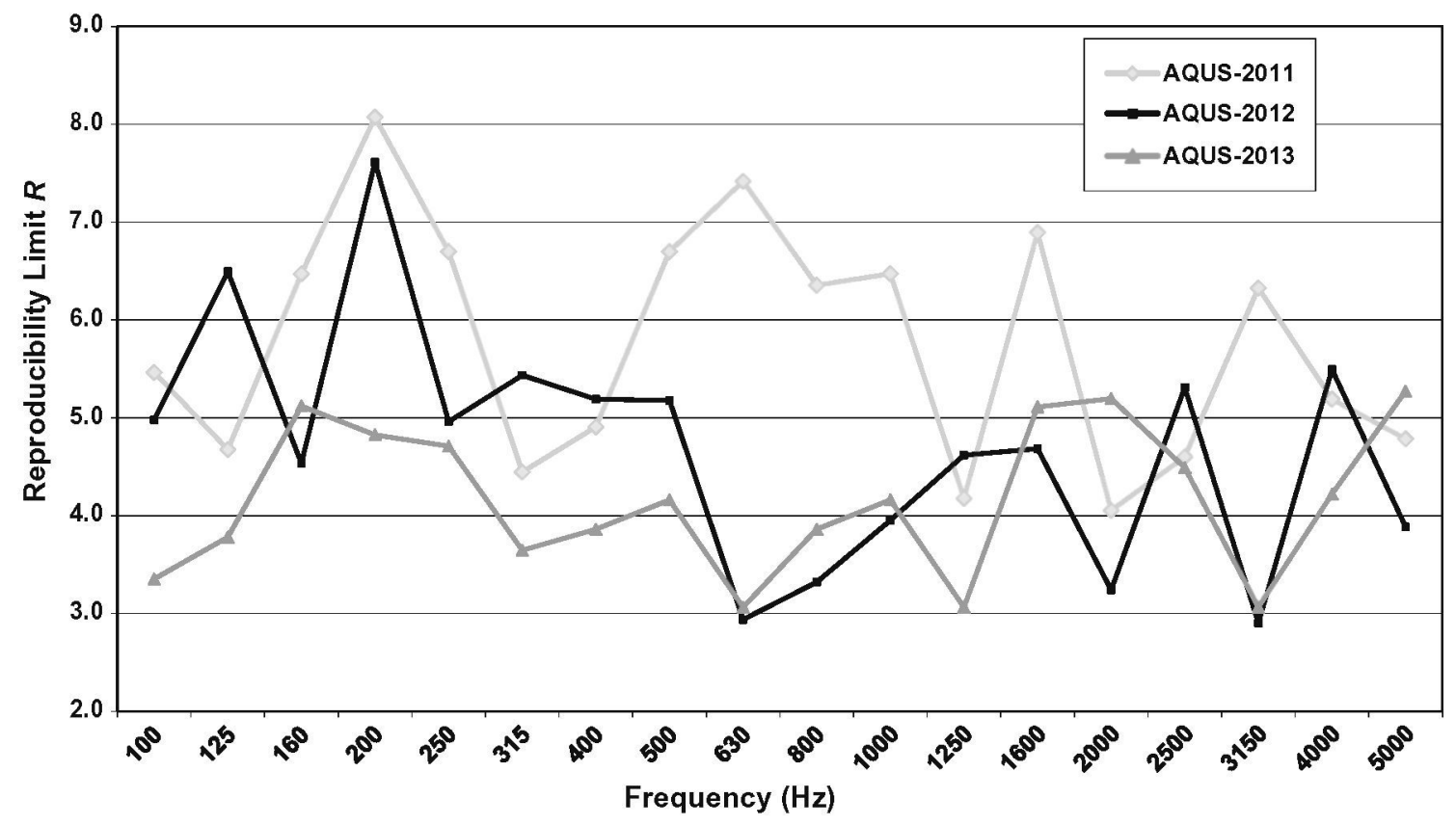

Fig. 2 Comparison of reproducibility limits $R$ obtained in AQUS program (years 2011-2013)

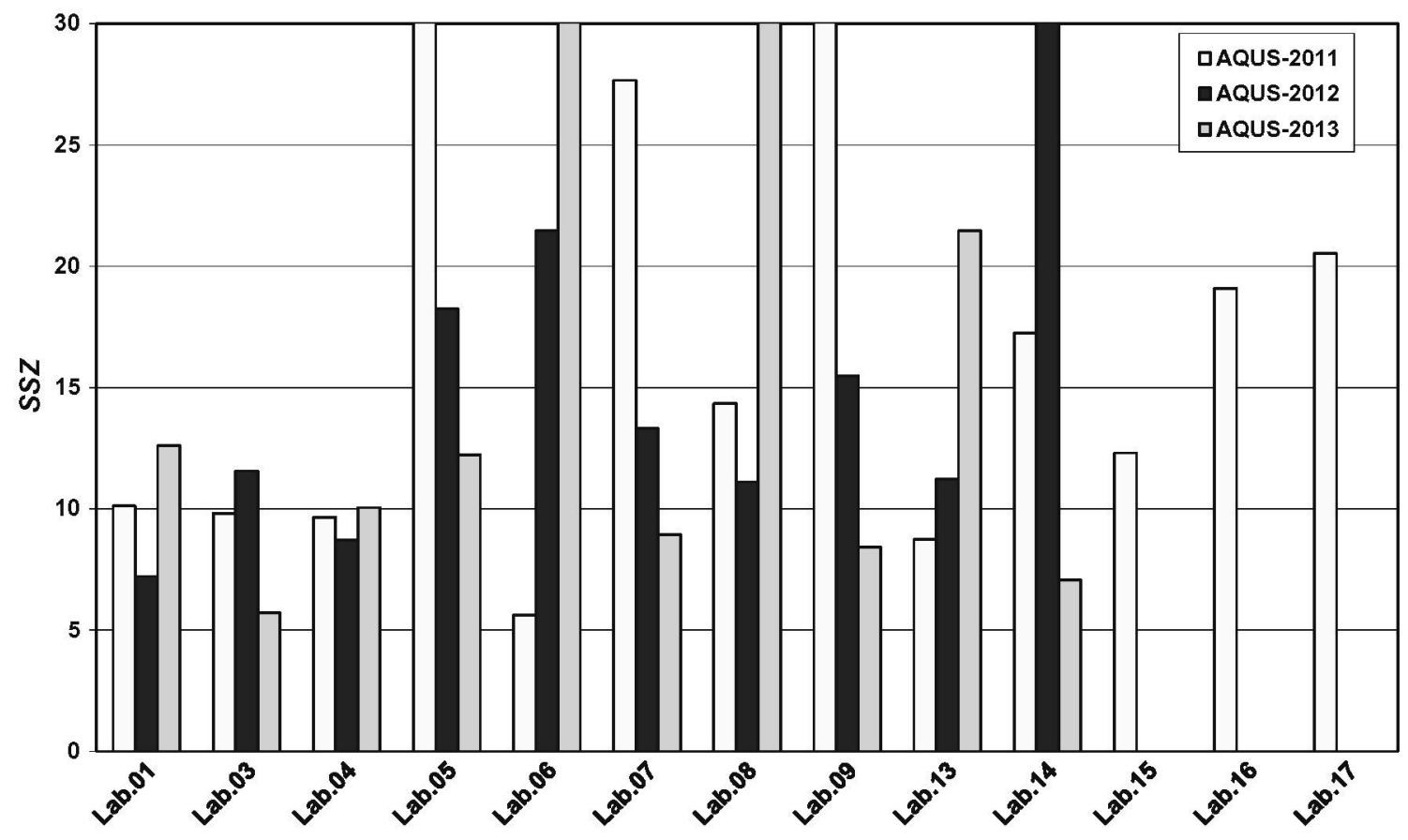

Participant

Fig. 3 Evolution of SSZ combined performance scores among the participants

As it can be noticed, since the sound pressure levels measured inside the building element are usually more widely spread at low signal frequencies due to a less uniform sound diffusion, the proposal $R$ values below $200 \mathrm{~Hz}$ are greater than the ones for mid-frequencies. Furthermore, the higher the frequency, the more directional is the sound source, so a slight variation in the incidence of the source directed to the facade makes more critical the insulation 
measurement above $3000 \mathrm{~Hz}$. Accordingly, at high frequencies, it is expected that the $R$ values might increase.

It is important to declare that these simulated reproducibility limits should be considered just as a mere probationary proposal based on experimental data (Fig. 4), so in the future those figures must be confirmed in a dedicated collaborative trial in a controlled scenario [11] with experimental conditions according to the information derived from existing practice.

\section{Conclusions and future objectives}

The next steps for the completion of the activities related to the present project should be aimed at the development of a collaborative study for the assessment of the performance of the measuring method, which makes the establishment of precision guidelines possible from the experience acquired in the field of measurements carried out according to

Table 2 Expert laboratories in terms of SSZ values over the rounds

\begin{tabular}{llll}
\hline Participant & AQUS-2011 & AQUS-2012 & AQUS-2013 \\
\hline Lab. A & 10.12 & 11.56 & 10.05 \\
Lab. B & 5.63 & 7.21 & 12.60 \\
Lab. C & 14.34 & - & 8.94 \\
Lab. D & 12.31 & 11.11 & 5.71 \\
\hline
\end{tabular}

ISO $140-5$ (under revision by ISO 16283-3). It is expected that the study will enable to assess if the prescribed reproducibility values for each frequency are suitable in terms of minimum precision level to determine the standard deviation for proficiency assessment.

Therefore, in this paper, it is proposed an approach based on the development of a collaborative test in controlled conditions, focused on the method rather than on the laboratory. Some points of interest that must be foreseen are related to both the applicability and practicability of the method since a collaborative trial requires substantial effort and should only be applied to methods that have received adequate prior testing.

The goal is to provide reliable and updated criteria for the evaluation of repeatability and reproducibility values in the measurement of sound insulation in buildings and building elements for facades, particularly appealing in those scopes in which nowadays there is no normative reference or this is very poor. In this way, by accomplishing this collaborative trial, future participants might have some kind of reference values available for the assessment of the precision criteria in this field. Furthermore, these prescribed values of precision should be verified regularly, as well as monitoring the practical conditions of application in such a way that the reproducibility values may also be used to verify the proper operation of test procedures of any laboratory involved in this scope of acoustic measurement.

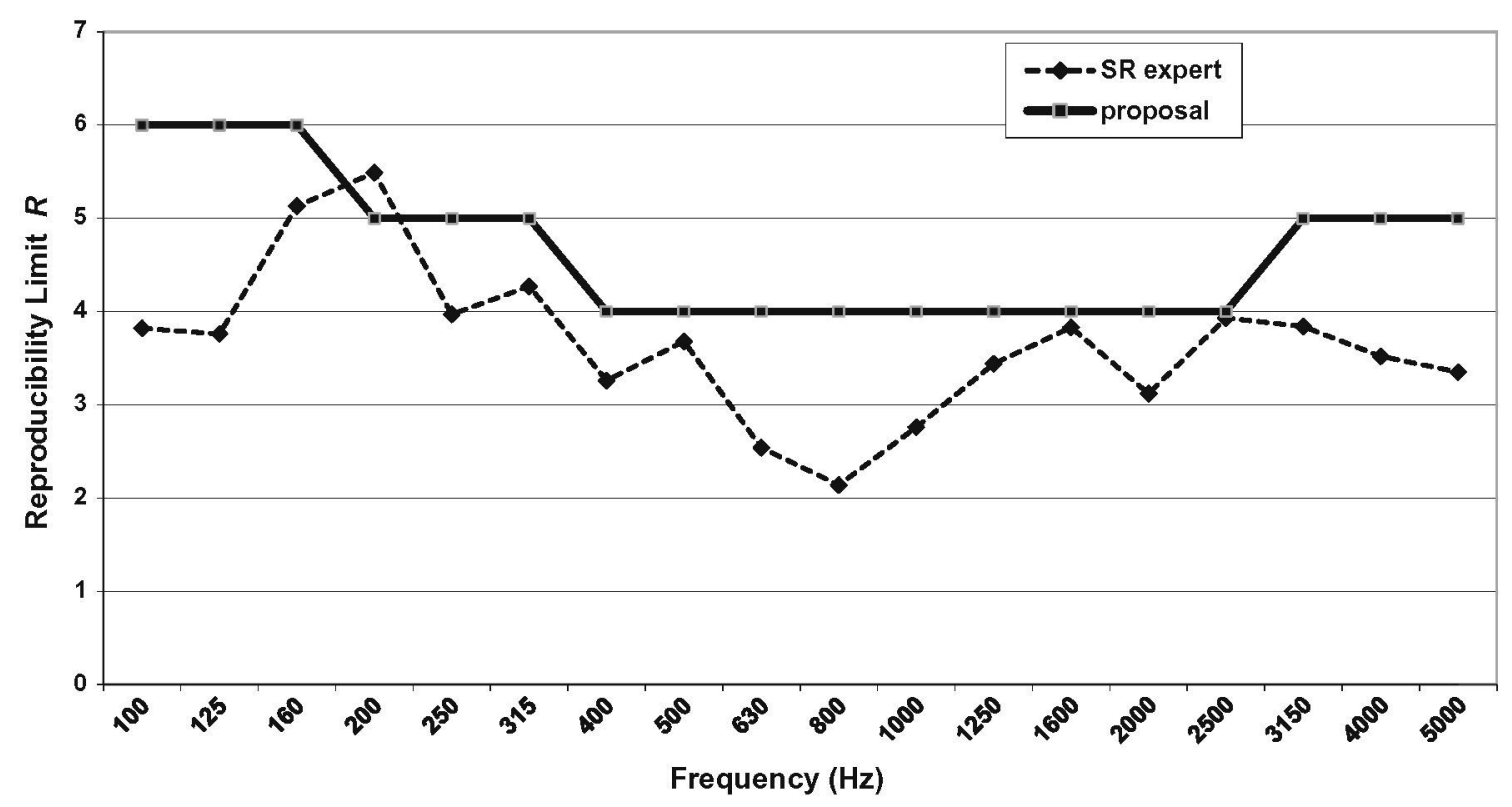

Fig. 4 Proposal on reproducibility limits for $D_{1 \mathrm{~s}, 2 \mathrm{~m}, \mathrm{nT}}(100-5000 \mathrm{~Hz})$ from expert laboratories performance over time 


\section{References}

1. ISO 140-5:1998 Measurement of sound insulation in buildings and of building elements-Part 5: Field measurements of airborne sound insulation of facade elements and facades. International Organization for Standardization, Geneva, Switzerland

2. ISO 17025:2005 General requirements for the competence of testing and calibration laboratories. International Organization for Standardization, Geneva, Switzerland

3. ISO 717-1:2013 Acoustics-rating of sound insulation in buildings and of building elements-Part 1: Airborne sound insulation. International Organization for Standardization, Geneva, Switzerland

4. Farina A, Fausti P, Pompoli R, Scamoni F (2006) Intercomparison of laboratory measurements of airborne sound insulation of partitions for the determination of repeatibility and reproducibility values. Istituto di Ingegneria, University of Ferrara, Ferrara

5. www.acustilab.es/.../Informe Final Global AQUS 2013 3 Ver_1. pdf

6. ISO 5725-2:1994 Accuracy (trueness and precision) of measurement methods and results-Part 2: Basic method for the determination of repeatability and reproducibility of a standard measurement method. International Organization for Standardization, Geneva, Switzerland

7. ISO 140-2:1998 Measurement of sound insulation in buildings and of building elements-Part 2: Determination, verification and application of precision data. International Organization for Standardization, Geneva, Switzerland

8. ISO 13528:2005 Statistical methods for use in proficiency testing by interlaboratory comparisons. International Organization for Standardization, Geneva, Switzerland

9. Ivanov V, Diaz C, Pedrero A, Sanchez J (2011) Influence of loudspeaker directivity on the measurement uncertainty of the acoustic testing of facades. International Congress on Noise Control Engineering. http://oa.upm.es/7884/1/INVE_MEM_ 2010_79330.pdf

10. Côté I, Robouch P, Dumas P, Pedneault M et al (2012) Determination of the standard deviation for proficiency assessment from past participants performance. Accred Qual Assur 17:389-393

11. Miller JC, Miller JN (1988) Statistics for analytical chemistry. Ellis Horwood Limited, Chichester, pp 85-91 